\title{
Challenges for efficient health service delivery: experiences of dietitians completing their compulsory community service year in South Africa
}

\author{
Whadi-ah Parker ${ }^{1, *}$, Nelia P Steyn ${ }^{1}$, Zandile Mchiza ${ }^{1}$, Edelweiss Wentzel-Viljoen ${ }^{2}$, \\ Andre Dannhauser ${ }^{3}$, Xikombiso Mbhenyane ${ }^{4}$, Gladys Nthangeni ${ }^{5}$ and Lynn Moeng ${ }^{6}$ \\ ${ }^{1}$ Centre for the Study of the Social and Environmental Determinants of Nutrition, Population Health, Health \\ Systems and Innovation, Human Sciences Research Council, Plein Park Building, Plein Street, Private Bag \\ X9182, Cape Town 8000, South Africa: ${ }^{2}$ Centre of Excellence for Nutrition, Faculty of Health Sciences, North \\ West University, Potchefstroom Campus, Potchefstroom, South Africa: ${ }^{3}$ Department of Nutrition and Dietetics, \\ School for Allied Professionals, Faculty of Health Sciences, University of Free State, Bloemfontein, South Africa: \\ ${ }^{4}$ Nutrition Department, University of Venda, Limpopo, South Africa: ${ }^{5}$ Department of Correctional Services, \\ Pretoria, South Africa: ${ }^{6}$ Directorate Nutrition, National Department of Health, Pretoria, South Africa
}

Submitted 20 July 2011: Accepted 9 November 2011: First published online 8 December 2011

\begin{abstract}
Objective: To evaluate the successes and shortcomings of the community service programme implemented by the Department of Health (DOH) in South Africa by evaluating community service dietitians' experiences and challenges during the 2009 community service year.

Design: The study employed both quantitative and qualitative research designs. A national survey was conducted using a questionnaire to illicit information on community service dietitians' working environments and the challenges they experienced. Furthermore individual interviews were conducted with a purposively selected subsample of community service dietitians to further explore issues that arose in the survey.

Setting: Data were collected from community service dietitians in the public health sector in South Africa.

Subjects: Dietitians completing their community service year in 2009.

Results: Of the 168 community service dietitians placed in 2009, 134 (80\%) participated in the survey, while five community service dietitians in each province ( $n$ 45) were interviewed. Overall the community service dietitians were positive about the community service year and reported that it improved their confidence, skills and competencies. However, they experienced challenges related to the orientation programme, supervision/mentoring, resources available, patient referrals and communication.

Conclusions: Despite the overall success of the community service year there are still challenges which the $\mathrm{DOH}$ needs to address in order to provide the best nutrition service possible for its patients.
\end{abstract}

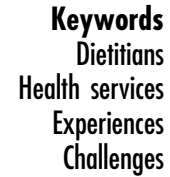

Keywords

alth services

Challenges
Nutrition-related disorders, many of which can be treated or prevented, contribute significantly to the overall burden of disease in South Africa. Treatment and prevention of these disorders are dependent not only on effective nutrition services but also on an overall efficient health system. In the World Health Report of 2000, the WHO stated that an efficient health system is dependent on three key inputs: human resources, physical capital and consumables $^{(1)}$. Based on these key inputs, the WHO drafted a framework identifying six components that would guide efforts to strengthen health systems. These components are: service delivery, workforce, information services, medical products and technologies, financing and leadership/governance ${ }^{(2)}$.

Although human resources are an essential component for the delivery of nutrition services, the nutrition workforce in South Africa is undoubtedly insufficient. By December 2008, only 1704 dietitians were registered with the Health Professions Council of South Africa (HPCSA), with less than 650 employed in the public health system. At the same time the South African population was an estimated 49 million $^{(3)}, 80 \%$ of whom rely on the public health sector, resulting in a ratio of 1:60308. Although South African universities have produced between 
147 and 230 newly qualified dietitians per annum since 2005, many of these nutrition professionals are lost to the public health system as they either opt to work in the private sector or migrate to developed countries where they believe their profession will be better rewarded ${ }^{(4)}$.

In order to address the shortage of human resources in South Africa, the Department of Health (DOH) instituted a compulsory community service (CS) policy for medical graduates in 1997. This was extended to include all allied health professionals (including dietitians) in 2002. This policy ensures that newly qualified health professionals complete a year of CS in the public health sector before they can register for independent practice with the HPCSA. Furthermore, it is aimed at ensuring that there is an equitable distribution of health professionals in underserved communities, particularly those in remote rural areas.

Studies on CS doctors, dentists, pharmacists, physiotherapists and clinical psychologists have shown that the CS programme has undoubtedly started to address both the workforce and service delivery components, by supplying health professionals to remote communities and to disadvantaged people. However, these health professionals continue to face challenges related to heavy workloads, a lack of resources and a lack of supervision ${ }^{(5-9)}$. It is therefore imperative that the financing, medical products and technologies, information systems and leadership/governance components of the WHO framework also be addressed.

With regard to nutrition services, two studies have been conducted on community service dietitians (CSD): a national mail survey in $2003^{(10)}$ and a provincial qualitative study in $2005^{(11)}$. The current survey, which was initiated and completed under the auspices of the Professional Board for Dietetics of the HPCSA, aimed to evaluate both CSD training and competencies (published elsewhere) as well as their experiences and the challenges they faced seven years post implementation of the CS policy. The present paper focuses on CSD experiences and challenges related to the components of the WHO framework as well as their suggestions for the $\mathrm{DOH}$ to improve both service delivery and the overall CS experience.

\section{Methods}

\section{Study design}

Quantitative methods in the form of a nationally representative cross-sectional descriptive design and qualitative methods in the form of individual interviews were employed.

\section{Population and sample selection}

The study population consisted of all CSD completing their compulsory CS year in South Africa in 2009. A list of CSD and their placements ( $n$ 168) were obtained from the Nutrition Directorate of the DOH. A subsample of forty-five CSD (five in each province) were purposively selected on the basis of the size (small or large) and location (urban, peri-urban (informal settlements) or rural) of the institution in which they were placed in order to cover a broad spectrum of experiences.

\section{Instruments, data collection and analysis}

Researchers developed a survey questionnaire to elicit information regarding the experiences and challenges CSD encountered in the CS setting. The questionnaire was validated in terms of face and content validity and pre-tested on a sample of dietitians ( $n$ 10) who had recently (2007 and 2008) completed their CS year. After minor corrections the questionnaire was delivered to CSD in the second half of 2009 at the health facilities where they were stationed, via post, email, fax or one of the researchers. Quantitative data were captured in a database (Microsoft ${ }^{\circledR}$ Access 2003; Microsoft Corporation, Redmond, WA, USA) and analysed using the SPSS statistical software package version 18 (SPSS Inc., Chicago, IL, USA).

In-depth interviews were held to further explore issues which arose from the quantitative study within the limits of the sample framework, in an attempt to reach data saturation. The interviews were conducted by trained field workers using a semi-structured interview schedule as a guide. Interviews were conducted telephonically or in person where possible and captured with the use of a tape/ digital recorder. All interviews were conducted in English or Afrikaans and transcribed verbatim. All transcripts underwent an initial quality assurance process. Afrikaans transcripts were then translated and back translated for a second round of quality assurance. Data were then transferred to the ATLAS.ti software program version 5 (2003-2011; ATLAS.ti Scientific Software Development GmbH, Berlin, Germany) for analysis. Data were analysed using a thematic content analysis approach. The transcripts were reviewed and the data were coded according to predetermined themes and categories.

\section{Etbical approval}

Ethical clearance was obtained by the Medical Research Council's Ethics Committee. Confidentiality was ensured as all questionnaires and interviews were completed and conducted voluntarily and returned and recorded anonymously.

\section{Results}

\section{Sample characteristics}

Of the 168 CSD placed in 2009, $80 \%$ ( $n$ 134) participated in the survey, while forty-five were purposively selected to participate in the qualitative interviews. The sample was well represented nationally (Fig. 1), with $55 \%$ placed in rural areas, $31 \%$ in urban areas and the remainder in urban informal settlements. The majority (64\%) indicated that they worked at district level while the rest were 


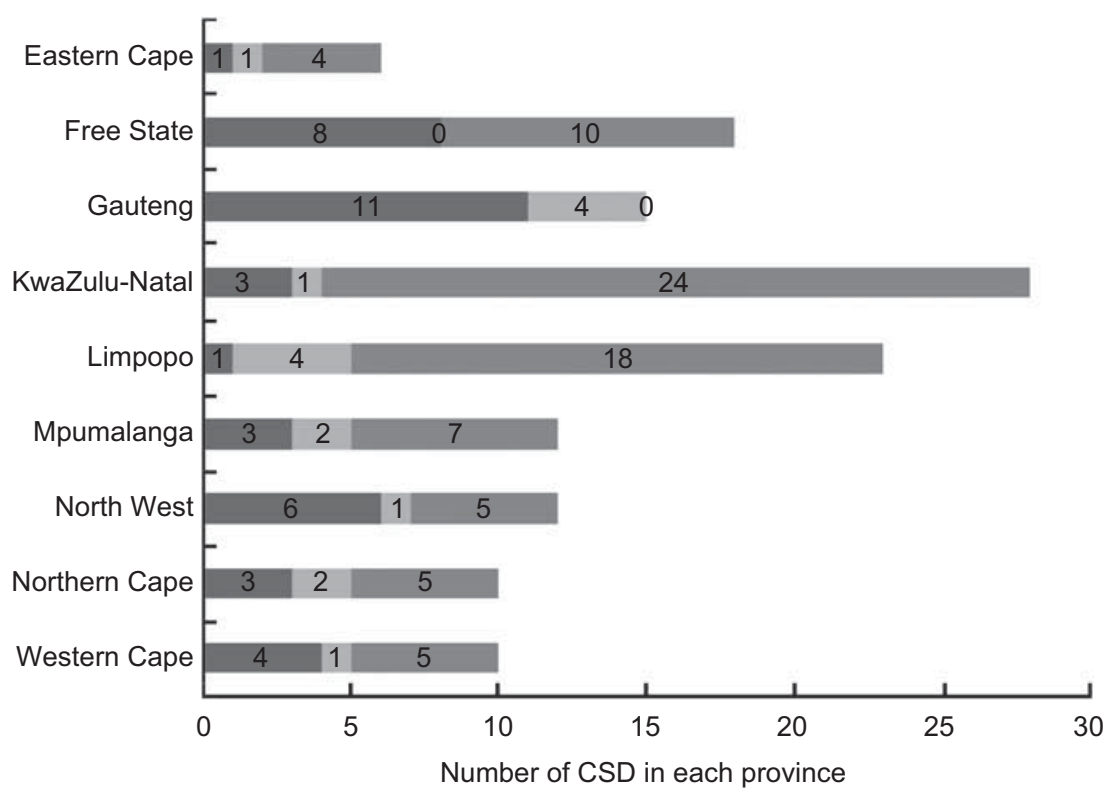

Fig. 1 Number of community service dietitians (CSD) completing their community service year in the nine provinces in South Africa in 2009 according to institution location ( $\square$, urban, $\square$, peri-urban (informal settlements); $\square$, rural)

equally distributed between primary health care (PHC) and tertiary facilities.

\section{Readiness of receiving institutions}

The survey results showed that $85 \%$ of CSD indicated health facilities were ready to receive them and $78 \%$ had a delegated office space. However, $20 \%$ were not allocated a working space and more than a third (38\%) felt that the space allocated was not adequate to do their work. Only $31 \%$ had their own email facility, of whom $59 \%$ indicated that it did not work most of the time. Similar results were reported for Internet access.

\section{Experiences}

Overall, CSD experienced their CS year positively. More than $60 \%$ of those interviewed reported that they enjoyed the year. This response was consistent across all provinces, irrespective of urban/rural placement. Some CSD enjoyed the year to such an extent that they were hoping to stay on' at the same facility the following year, while others stated that completing a CS year was beneficial and urged the DOH 'to continue providing such a service' as it is an 'essential way to introduce graduates to how the DOH functions, how the structures work and what its priorities are'.

Survey results for positive aspects of CS are listed in Table 1. More than a third (34\%) indicated that they gained practical experience, including how to make do with limited resources and in terms of the systems and administration procedures of a government institution. Furthermore, $30 \%$ indicated that they experienced both professional development and personal growth. Qualitative results provide corroboration; for example, one CSD stated
Table 1 Positive experiences of community service reported by community service dietitians ( $n$ 134), South Africa, 2009

\begin{tabular}{lrr}
\hline Positive experiences & $n$ & $\%$ \\
\hline Gained practical experience & 45 & 34 \\
Experienced professional development and & 40 & 30 \\
$\quad$ personal growth & 27 & 20 \\
Personally rewarding & 21 & 16 \\
Interaction with the multidisciplinary team & 20 & 15 \\
Good supervision/support structure & 9 & 7 \\
Learning new cultures and languages & 7 & 5 \\
Exposure to all aspects of dietetics & 5 & 4 \\
Good remuneration & 8 & 6 \\
Provided exposure to community dietetics/outreach & & \\
$\quad$ services & 4 & 3 \\
Reduced anxiety about finding employment & 3 & 2 \\
Developed relationships with other health professionals & 3 & 2 \\
Opportunity to learn to work with limited resources & 2 & 1 \\
Opportunity to advocate the dietetics profession & 1 & 1 \\
Good systems & & \\
\hline
\end{tabular}

that she 'gained a lot of experience and confidence in terms of management and organising the nutrition programme'. Of the forty-five CSD interviewed, twenty-nine indicated that CS provided an overall learning experience, some of whom stated that working with 'senior dietitians and fellow CSDs that studied at different training institutions' in conjunction with 'being a part of the multidisciplinary team' contributed to the overall learning experience of the CS year.

Twenty per cent indicated that it was personally rewarding to provide services where they were most needed as CS provided exposure to outreach services. While $7 \%$ reported that CS provided them with an opportunity to learn about new cultures and languages, it also afforded them the opportunity to advocate the dietetics profession: one CSD 
Table 2 Problems related to community service placements as reported by community service dietitians (n 134), South Africa, 2009

\begin{tabular}{lcr}
\hline Problems cited & $n$ & $\%$ \\
\hline Limited resources & 33 & 25 \\
Lack of supervision and guidance and support & 19 & 14 \\
Staff shortage & 13 & 10 \\
Lack of referrals & 11 & 8 \\
Placement process and orientation & 11 & 8 \\
Establishing and marketing a department/administration and systems & 11 & 8 \\
Lack of acceptance and acknowledgement of the dietetics profession & 9 & 7 \\
Accommodation & 8 & 6 \\
Location of placement & 7 & 5 \\
Self-confidence and time management & 3 & 2 \\
\hline
\end{tabular}

stated that it created awareness of the role of a dietitian within the community since the 'community can now see where nutrition comes into their daily lives'.

Other positive aspects of CS included good supervision and support structures (15\%) and to a lesser extent, exposure to all aspects of dietetics (5\%), good remuneration (4\%) and reduced anxiety about finding employment (3\%).

\section{Challenges}

Table 2 provides a summary of the challenges that CSD reported during the survey. It is interesting to note that $9 \%$ of CSD reported they experienced no challenges during their CS year. Furthermore, those who indicated experiencing challenges stated that most challenges improved with time.

The most common challenge was related to limited resources (25\% in the survey and $40 \%$ in the interviews). More than half of the CSD were placed in rural areas, in small under-resourced hospitals. During the interviews, twelve CSD raised the issue of financial restrictions with a resultant scarcity of nutritional supplements. For example, these CSDs stated 'I don't have a budget so I can't order the feeds that I need' and 'I don't have porridge to give to the patients with BMI's below 18.5 but you have to make do with what you have'. The lack of nutritional supplements directly hampers service delivery to both inpatients and out-patients. During the interviews, fourteen CSD suggested securing larger budgets for nutritional supplements which would enable them to buy better formula for the babies and rehabilitate them in a more efficient way' and to improve the hygiene associated with mixing feeds by 'replacing powder feeds with ready-touse feeds because they're not preparing the powder feeds as they should and there's lots of cross contamination'.

According to the survey, $78 \%$ of CSD had a delegated office space; however, during the interviews it became clear that those who did not experienced dire challenges in this regard. One CSD stated: 'without an office I can't do proper counselling ... I don't have any privacy with patients ... neither do I have a proper storeroom to store supplements'. Three CSD therefore suggested that the DOH should ensure they have sufficient office space and basic office equipment (desks, computers and printers). Similarly, they suggested that if the DOH places a dietitian in the community, they should ensure that she has basic equipment such as a scale, a glucometer and supplements at her disposal'.

Furthermore, CSD are expected to travel to surrounding clinics in order to provide outreach services. However, this service is dependent on adequate transport to and from these facilities, which is usually shared among all allied health professionals. One CSD stated that this often compromises patient care and service delivery as they make us wait for the physiotherapist and the occupational therapist when I could've been back at the hospital seeing patients'. She therefore suggested that by 'improving planning and distribution of resources it would be easier for us to see more patients and improve patient care'.

As many as $20 \%$ of CSD, during both the interview (11/45) and survey (24/134), reported challenges related to language and communication. Issues related to language barriers have been reported and discussed elsewhere. In terms of communication CSD suggested that the $\mathrm{DOH}$ (at a district or provincial level) holds quarterly meetings with all CSD post orientation in order to follow up on difficulties or concerns that arise. They also raised the need to improve 'communication between the different programmes and coordinators (within the DOH) since they are supposed to work together; but they don't communicate'. In addition, in order to promote the role of the CSDs and promote awareness of what is occurring in the hospital, they suggested that meetings should be held regularly to share information in such a way that health professionals from various disciplines are made aware of what their colleagues are doing.

There were also reports of a lack of support from management. One CSD stated that although her direct 'supervisor within the hospital is supportive; sometimes there are things that he cannot agree to on his own and needs to put forward to the management committee who disagree with everything'. As a result, CSD suggested that the DOH should encourage management to be supportive of CS professionals in order to facilitate a positive working environment. They further stated that too often 
hospital management is not informed in a timely way about the placement of CS professionals and as a result the hospital is unprepared for their arrival. They therefore suggested it is vital that the DOH communicates directly with the management of the institution prior to the placement of CS professionals such that 'CEOs and the hospital managers know exactly who is placed there, what their role is and what is expected of them, that way they will know exactly what they should be doing for us and what we should be doing for them'.

Survey results showed that $58 \%$ of CSD indicated their line manger was a dietitian, $23 \%$ and $6 \%$ were supervised by a doctor or a nurse, respectively, and $17 \%$ were supervised by other health professionals. Although all CSD had some form of supervision, only $65 \%$ indicated that they had adequate supervision, while $22 \%$ and $13 \%$, respectively, indicated that they had adequate supervision some of the time or hardly ever. Furthermore, $14 \%$ of CSD reported that they experienced the lack of supervision and support as a challenge. These reports were often made by CSD who were placed in institutions where there were previously no dietetic services; however, one CSD stated that she learned a lot from not having a supervisor since I had to do most things on my own'.

Overall though, during the interviews, five CSD reported that it would have been easier to adjust if someone showed them around within the first two weeks. Although there were mentors, they were often not located at the hospital and thus were unable to provide hands-on assistance. For these reasons they suggested that the DOH should implement a handover period of approximately two weeks where 'the old and new CSDs overlap a little bit'. Where this was not possible they suggested that a handover file, 'a precise document on what is supposed to happen', be developed and made available to the new CSD. Further suggestions included implementation of an effective mentoring system where the mentor should be appropriately selected and should be based at the health facility.

In the survey, $10 \%$ of CSD highlighted challenges related to the staff complement and their resultant workloads. Nine of the CSD interviewed corroborated this result, with statements like 'we were three dietitians for a nine hundred-bed hospital ... of which two were CSDs' and 'I was the only dietitian in the hospital ... it's not a big hospital but I'm also responsible for all the surrounding clinics'. In addition, three CSDs raised the issue of the lack of continuity of dietetics services between consecutive CS years: 'as CSDs come and go, the projects and programmes they try to put in place are not continued'. Furthermore they stated that the duration of community service may be too short since it takes a few months to get used to the working world, only by June/July do you feel confident to start initiating projects but by then it's almost the end of the year and you have to start wrapping up, so you don't really have enough time to follow through on what you initiated'.
Survey results further showed that only $4 \%$ of CSD indicated their role as a dietitian was hardly ever understood by the health team, while $75 \%$ and $25 \%$ of CSD reported being accepted by the multidisciplinary team most times and sometimes, respectively. However, $7 \%$ of CSD reported that the dietetics profession is still not receiving adequate recognition for its role in overall health and well-being. This is substantiated in CSD responses related to patient referrals, where only $60 \%$ and $32 \%$ of CSD reported receiving referrals from health team members most times or sometimes, respectively. For these reasons ten CSD who were interviewed suggested that the DOH should advocate both 'role of nutrition therapy in the total well-being of a patient' and 'the role of a dietitian since some communities don't know what a dietitian is'.

CSD who were placed in institutions where there were previously no dietetics services reported that establishing and marketing a dietetics department was a substantial challenge ( $8 \%$ of those surveyed). These results were corroborated by four CSDs during the interviews: 'I had to liaise with all the core departments and get to know everyone and get them to refer patients to me'. Furthermore, during the interviews ten CSD reported that they experienced difficulty with familiarising themselves with the administration procedures and systems followed in a government institution: 'learning the procedures and policies were quite a challenge ... how the government works, how the systems work, how to order supplements'.

During the interviews, six CSD also highlighted challenges related to their orientation programme, such as the timing: 'orientation took place in May/July ... it should be in January/February so that we can be aware of what programmes are running and what is expected of us and what we can expect of them'; as well as the content of the orientation programme: 'orientation is usually limited to 3 days and it's impossible to cover everything ... but they should create awareness of things that we were not previously exposed to like employee performance systems and completion of administrative forms'.

Finally, with respect to placement, four CSD suggested that they should be able to select their placement based on the area in which they want to specialise, albeit therapeutic, community or food service in nature, while others requested that they be placed in bigger institutions 'where we could learn more' or 'if possible, that every CSD rotate between a tertiary provincial hospital and a community placement'.

\section{Discussion and recommendations}

CS was implemented by the DOH with the aim of delivering services to previously underserved communities. The results of the present study show that the $\mathrm{DOH}$ is succeeding in this regard since the majority of CSD were placed in rural and peri-urban communities: mainly at district and PHC 
institutions, with a large proportion regularly involved in outreach services, thereby providing services where they previously were not available.

Previous authors have reported that the majority of CS officers felt their experience had been personally and professionally rewarding and that they provided a good service, did worthwhile work and made a difference in the community ${ }^{(8-10,12,13)}$. The results of the current study echo these findings as the vast majority of CSD reported that they enjoyed their year as it provided them with good work experience and opportunities for personal growth and self-confidence.

Although many positive elements regarding CS have been reported, these are inevitably accompanied by numerous challenges, some of which are specifically related to leadership and governance in public health institutions. Previous studies have stated that CS was initially implemented without adequate preparation of both receiving institutions and CS professionals ${ }^{(14)}$. Seven years later, this problem still exists, albeit to a far lesser degree. CSD in the current study still reported that receiving institutions were not entirely prepared for their arrival such that some CSD were not allocated a working space, and those who were allocated space often reported that it was inadequate. It is therefore critical that the $\mathrm{DOH}$ provides receiving institutions with information regarding the allocation of CS officers in a timely way.

Paterson et al. ${ }^{(11)}$ stated that CSD in KwaZulu-Natal were unprepared for CS. This is reflected nationally since CSD in the present study reported that there is confusion regarding their job description. What is even more concerning is the fact that this uncertainty is not limited to the CSD, but extends to the level of the receiving institutions as well as the district offices. Furthermore some CSD are not entirely aware of the overall aim of the CS programme, which, as stated previously, is to provide services to underserved communities. This became abundantly apparent when CSD in the current study suggested that they be placed in larger institutions where they could gain exposure to a wider variety of therapeutic experiences and improve their professional development. It is therefore crucial for both the $\mathrm{DOH}$ and the training institutions to clarify the difference between an internship and a CS placement. It is also crucial that the CS programme be properly defined to all concerned (students, hospital staff and management at district and provincial level) and that what is expected of CSD, whether it is therapeutic, community or food service related, is clarified prior to their placement. In this regard the Nutrition Directorate of the DOH could provide guidelines for universities to inform future CSD of the nature of CS placements. Alternatively the same guidelines can be used during CS orientation. Furthermore, for this reason it is essential that CS orientation programmes take place earlier in the year (January or February) and not in May or June as was the case.

Another challenge related to leadership/governance centres on supervision of and support for CSD. Although there is a mentoring system in place, the lack of supervision and support experienced on site appears to be a major challenge for CSD. In the current scenario nearly $60 \%$ of CSD were directly supervised by a dietitian, while some were supervised by a doctor or other health professional. A large percentage however felt that they did not have adequate mentoring. In this regard the research undertaken in KwaZulu-Natal ${ }^{(11)}$ reflects the underlying perceptions and feelings that having a dietitian (as a line manager and mentor) is essential in order for professional development to take place, since practice on its own, without input to improve weaknesses, will result in failure. Couper ${ }^{(14)}$ described a programme to develop mentors for CS doctors in northern KwaZulu-Natal in which a group of senior doctors formed a team of mentors to new CS doctors at district hospitals. This was shown to provide many benefits for their professional development and provides an important model for mentorship in the public health sector. However, it is dependent on having a senior doctor present at the same facility as the CS doctor. Although this may not be possible with dietitians at every facility, it may be possible to set up mentoring groups at district level and this is certainly encouraged. Alternatively, Couper ${ }^{(14)}$ also stated it is important that health professionals at receiving institutions as well as CSD view themselves as 'just another dietitan'. In this way CSD will be viewed, accepted and ultimately perform as fellow health professionals, and not as students who require a large amount of supervision and support.

In terms of challenges related to financing and medical equipment, previous studies conducted on CS dentists, doctors and clinical psychologists indicated that access to equipment and drugs was pivotal in enabling them to provide basic services $^{(8,9,13)}$. Fortunately, CSD are in a better position since they do not require specialised equipment in order to provide basic services to their clients. However, in order to function optimally CSD do require resources such as nutritional supplements and nutrition education materials which were reportedly in short supply as a result of financial restrictions at receiving institutions. Dietetics departments within public health institutions should thus receive adequate financial resources to operate optimally.

Apart from requesting that systems be implemented to standardise the recording of patient statistics, CSD in the current study did not raise many challenges related to information services. What they did raise were challenges related to communication, which were also reported by Paterson et $a l^{(11)}$. These included the lack of understanding of the role of a CSD by other health professionals, the lack of recognition of their profession and the underutilisation of their services. It is therefore crucial that the DOH promotes and advocates the role of the dietitian, as well as the role of nutrition therapy, in overall patient care. 
Although the CS policy begins to address workforce challenges in the public health sector it is important to realise that CS is essentially an effective recruitment strategy; but it is by no means a retention strategy. Ross and Reid ${ }^{(13)}$ stated that by the end of each year, most CS professionals are skilled, valued and valuable members of the health team who are able to cope with the demands of working in the public health service within the resources available at district hospitals. Khan et al. ${ }^{(12)}$ reported that the proportion of CS therapists who indicated they would work in the public health sector declined from $50 \%$ at onset to $35 \%$ at exit and even fewer (24\%) said they would work in a rural area. Ross and $\operatorname{Reid}^{(13)}$ found that only $8 \%$ of officers, who completed their CS in 2002, remained at the same district hospital in 2003. Furthermore only $16 \%$ of CS officers in 2003 indicated that they were willing to remain at the same institution after completion of their CS year. CS officers were more likely to remain at their institution when they had been allocated to their first choice of placement (they are able to nominate three) and they were more likely to remain in a district hospital if they had grown up in a rural area. Placement of CS officers thus has a large impact on their retention.

Other factors associated with the retention of staff included improving orientation, mentoring, teamwork, professional development or promotional opportunities, medical equipment and accommodation ${ }^{(8-13)}$. Although retention figures are not available for dietitians, it is encouraging to note that CSD in the current study indicated willingness to remain at the CS placement if permanent posts were made available and there were opportunities for professional development. They were also more likely to remain if their placement was in the professional area they wanted to specialise in. Paterson et al. ${ }^{(11)}$ provided similar results. Thus if the DOH aims to retain staff who have completed CS, not only should more permanent posts be made available, but also more attention should be given to the placement process: where possible, CSD should be placed at the institution identified as their first choice and also within a position that complements the area in which they want to specialise.

In the absence of retaining CS personnel, yet another factor to consider is the lack of continuity of services between CS years. CSD suggested that, where possible, outgoing CSD provide a handover file for new CSD to facilitate continuity of services and orientation in their new environment. Paterson et al. ${ }^{(11)}$ reported similar findings related to a procedure manual containing relevant administrative information that had been compiled by CSD in the previous year. There was also a suggestion from CSD in the current study for the $\mathrm{DOH}$ to provide an overlapping period between outgoing and incoming CSD that would facilitate a more effective orientation and handover period. The $\mathrm{DOH}$ should thus address this issue by requesting outgoing CSD to provide these handover files and where possible amend the duration of CS to facilitate an overlap between incoming and outgoing CSD.

\section{Conclusions}

While the DOH is urged to continue to drive the CS agenda, it should take heed of the recommendations related to the following four components of the WHO framework to strengthen health systems: leadership/governance, financing, information services and retention of the workforce. Recommendations that should receive immediate attention are those related to clarifying the difference between an internship and a CS placement; timely provision of information regarding CS allocations to receiving institutions; and advocacy of the role of the dietitian and nutrition therapy in patient care.

\section{Acknowledgements}

This research was supported by the Health Professionals Council of South Africa, the Human Sciences Research Council and the South African Medical Research Council. The authors have no conflicts of interest. W.P., N.P.S., X.M., G.N. and L.M. contributed to the conception and design of the study. W.P., Z.M., E.W.-V., A.D., X.M. and G.N. were responsible for the acquisition of data. W.P. and N.P.S. were responsible for responsible for the analysis and interpretation of the data. W.P., N.P.S. and Z.M. were responsible for drafting the manuscript. W.P., N.P.S., Z.M., E.W.-V., A.D., X.M., G.N. and L.M. contributed to critical revision of the manuscript. The authors would like to acknowledge the contributions of Mr Vaughn Leiberum in data analysis and Ms Natasha Saunders in data capturing.

\section{References}

1. World Health Organization (2000) World Health Report 2000. Health Systems: Improving Performance. Geneva: WHO; available at http://www.who.int/whr/2000/en/ whr00_ch4_en.pdf

2. World Health Organization (2007) Everybody's Business Strengthening Health Systems to Improve Health Outcomes. WHO's Framework for Action. WHO: Geneva; available at http://www.who.int/healthsystems/strategy/everybodys_ business.pdf

3. Statistics South Africa (2010) Mid-year population estimates 2010. http://www.statssa.gov.za/publications/P0302/P0302 2010.pdf (accessed June 2011).

4. Kabene SM, Orchard C, Howard JM et al. (2006) The importance of human resources management in health care: a global context. Hum Resour Health 4, 20.

5. Sanders D \& Lloyd B (2005) Human resources: international context. In South Africa Health Review, pp. 76-87 [P Ijumba and P Barron, editors]. Durban: Health Systems Trust; available at http://www.hst.org.za/sites/default/files/sahr05_chapter6.pdf

6. Futter MJ (2003) Developing a curriculum module to prepare students for community-based physiotherapy rehabilitation in South Africa. Physiotherapy 89, 13-24.

7. Ramklass SS (2009) An investigation into the alignment of a South African physiotherapy curriculum and the expectations of the healthcare system. Physiotherapy 95, 216-223.

8. Reid S (2002) Community service for health professionals: human resources. In South African Health Review, pp. 135-160 [P Ijumba and P Barron, editors]. Durban: Health Systems Trust; available at http://www.hst.org.za/sites/ default/files/chapter8.pdf 
9. Pillay AL \& Harvey BM (2006) The experiences of the first South African community service clinical psychologists. S Afr J Psychol 36, 259-280.

10. Visser J, Marais M, du Plessis J et al. (2006) Experiences and attitudes of dietitians during the first compulsory community service year. S Afr J Clin Nutr 19, 10-17.

11. Paterson M, Green M \& Maunder EM (2007) Running before we walk: how can we maximise the benefits from community service dietitians in KwaZulu-Natal, South Africa? Health Policy 82, 288-301.
12. Khan NB, Knight S \& Esterhuizen T (2009) Perceptions of and attitudes to the compulsory community service programme for therapists in KwaZulu-Natal. $S$ Afr $J$ Commun Disord 56, 17-22.

13. Ross A \& Reid S (2009) The retention of community service officers at district hospitals in KwaZulu Natal, Eastern Cape and Limpopo Provinces. S Afr Fam Pract 51, 249-253.

14. Couper I (2004) Developing mentors for community service doctors. An experience from Northern KwaZuluNatal. $S$ Afr Fam Pract 46, 34-35. 